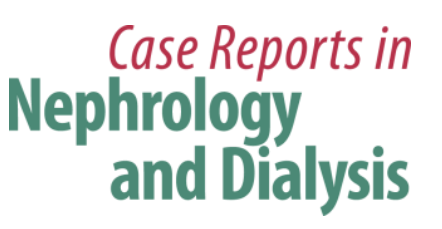

Case Rep Nephrol Dial 2019;9:8-14

DOI: 10.1159/000498844

Published online: February 28, 2019

(c) 2019 The Author(s)

Published by S. Karger AG, Basel

www.karger.com/cnd

This article is licensed under the Creative Commons Attribution-NonCommercial 4.0 International License (CC BY-NC) (http://www.karger.com/Services/OpenAccessLicense). Usage and distribution for commercial purposes requires written permission.

\title{
Monotypic IgG1-kappa Atypical Anti-Glomerular Basement Membrane Nephritis: A Case Report
}

\author{
Maxim Olivier ${ }^{\mathrm{a}}$ Harold Watson $^{\mathrm{a}}$ Danielle Lee $^{\mathrm{a}}$ Viresh Mohanlal $^{\mathrm{c}}$ \\ Mario Madruga ${ }^{a}$ Steven Carlan ${ }^{b}$ \\ aDepartment of Medicine, Orlando Regional Healthcare, Orlando, FL, USA; \\ ${ }^{b}$ Division of Academic Affairs and Research, Orlando Regional Healthcare, Orlando, FL, USA; \\ 'Division of Nephrology, Orlando Regional Healthcare, Orlando, FL, USA
}

\section{Keywords}

Renal failure $\cdot$ Atypical anti-glomerular basement membrane $\cdot$ Nephritis $\cdot$ Mycophenolate mofetil

\begin{abstract}
Anti-glomerular basement membrane (anti-GBM) glomerulonephritis is a rare disease caused by autoantibodies against the glomerular basement membrane. Atypical anti-GBM nephritis is clinically less aggressive and characterized by the absence of circulating autoantibodies to the basement membrane. A previously healthy 53 -year-old white woman presented with a rising creatinine over a short observation period. Renal biopsy, urinary sediment, and laboratory testing confirmed the diagnosis of atypical anti-GBM disease. She received plasmapheresis, steroids, and cyclophosphamide. She developed hemorrhagic cystitis early in the treatment from oral cyclophosphamide and mycophenolate mofetil was substituted as a first-line drug. She responded favorably and continued on mycophenolate mofetil without evidence of relapse. Despite the absence of circulating autoantibodies, a diagnosis of atypical anti-GBM nephritis should not be excluded if a high index of clinical suspicion exists. Early renal biopsy should be considered. Mycophenolate mofetil may be a reasonable replacement for oral cyclophosphamide in the treatment of atypical anti-GBM disease when cyclophosphamide is contraindicated.




\section{Case Reports in Nephrology and Dialysis}

\section{Introduction}

Anti-glomerular basement membrane (GBM) antibody nephritis is a rare autoimmune disorder characterized by the presence of circulating anti-GBM antibodies and diffuse linear staining for immunoglobulins along the GBM detected through immunofluorescence microscopy [1]. Classic anti-GBM disease typically presents with rapidly progressive glomerulonephritis and most cases are dialysis dependent on diagnosis or have a fast progression to endstage renal disease despite intensive immunosuppressive therapy and plasmapheresis [2]. Pulmonary involvement can occur in up to half of the cases of classic anti-GBM disease and usually presents with pulmonary hemorrhage. Recently, atypical forms of the disease have been reported with a more indolent clinical course and no pulmonary involvement. Absent detectable circulating autoantibodies but intact linear deposition and staining of the GBM for immunoglobulin G (IgG) through immunofluorescence technique is the hallmark of the diagnosis for atypical cases $[3,4]$.

Early detection through renal biopsy is critical since aggressive therapy is likely to preserve renal function long-term and delay or decrease the need for maintenance dialysis. The proposed treatment strategy for both classic and atypical anti-GBM disease has remained unchanged for decades and consists of intensive plasmapheresis combined with prednisone and cyclophosphamide [5]. There is no established second-line treatment, only rare reports of success with mycophenolate mofetil in place of cyclophosphamide [6].

We present a case of indolent atypical anti-GBM disease with no detectable circulating anti-GBM antibody diagnosed by an early renal biopsy and characterized by a contraindication to cyclophosphamide use resulting in mycophenolate mofetil selection as the primary immunosuppressant.

\section{Case}

A 53-year-old white female with a past medical history of hypothyroidism was referred to a nephrologist after detecting a serum creatinine of $1.8 \mathrm{mg} / \mathrm{dL}$ on routine lab work. Initially this finding was thought to be transient; however, labs the following month showed serum creatinine increased to $2.4 \mathrm{mg} / \mathrm{dL}$. Her only medications included levothyroxine $0.088 \mathrm{mg}$, once daily) and an occasional over-the-counter nonsteroidal anti-inflammatory drug, which she was advised to discontinue. She denied any symptoms including dysuria, increased urinary frequency, hematuria, rashes, ulcers, or joint pains or recent respiratory infections. She denied hemoptysis, cough, or dyspnea. Physical examination was unremarkable without evidence of pulmonary pathology. Laboratory studies on admission 2 weeks later showed the following values: serum creatinine of $1.89 \mathrm{mg} / \mathrm{dL}$; albumin, $3.6 \mathrm{~g} / \mathrm{dL}$; hemoglobin, $9.8 \mathrm{~g} / \mathrm{dL}$; white blood cell count, $8.8 \times 10^{3} / \mu \mathrm{L}$; platelet count, $225 \times 10^{3} / \mu \mathrm{L}$. Urine studies revealed $3+$ occult blood, 11-30 red blood cells, and a protein/creatinine ratio of $655 \mathrm{mg} / \mathrm{g}$ creatinine (normal, 0-200). The glomerular filtration rate was $28 \mathrm{~mL} / \mathrm{min} / \mathrm{SA}$ (surface area). In addition, tests for complements, anti-DNA (deoxyribonucleic acid) antibody, ANA (antinuclear antibody), MPO (myeloperoxidase), c-ANCA (cytoplasmic antineutrophil cytoplasmic antibodies), p-ANCA (perinuclear antineutrophil cytoplasmic antibodies) (typical and atypical), anti-proteinase 3 antibody and SPEP (serum protein electrophoresis), and UPEP (urine protein electrophoresis) were all normal. Her GBM IgG antibody was negative and no monoclonal bands were detected on urine or serum IFE (immunofixation electrophoresis). Hepatitis B surface antigen and hepatitis $\mathrm{C}$ antibody were both negative. Because of the rapid deterioration of 


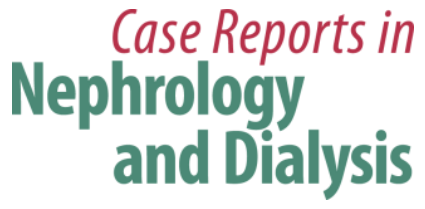

Case Rep Nephrol Dial 2019;9:8-14

DOI: $10.1159 / 000498844$

(c) 2019 The Author(s). Published by S. Karger AG, Basel www.karger.com/cnd

Olivier et al.: Atypical Anti-Glomerular Basement Membrane Nephritis

renal function, screening renal ultrasound was considered redundant and thus cancelled. Kidney biopsy revealed linear staining along the GBMs by monotypic IgG1-kappa (Fig. 1) with numerous red blood cell casts (Fig. 2), interstitial fibrosis, tubular atrophy, moderate arteriosclerosis, and moderate arteriolar hyalinosis of moderate chronicity. Electron microscopic examination of the glomerulus (Fig. 3) revealed widespread effacement of the foot processes and absence of any immune complex electron dense deposits. These microscopic findings along with serological results were diagnostic for atypical anti-GBM disease.

She was started on a 3-day regimen of intravenous methylprednisolone $500 \mathrm{mg}$ daily for 3 days then switched to oral prednisone $60 \mathrm{mg} /$ day, $150 \mathrm{mg}$ oral cyclophosphamide daily, and 5 sessions of plasmapheresis. Repeat serology was still negative. Pulmonary exams were consistently clear to auscultation without abnormalities and there were never any upper respiratory symptoms such as cough, hemoptysis, sputum production, or rhinitis. Thus, no chest imaging was done as it was not warranted. She was discharged from the hospital and 10 days into the treatment course, she developed hemorrhagic cystitis. Oral cyclophosphamide was discontinued and prednisone was reduced to $40 \mathrm{mg} /$ day. Mycophenolate mofetil $500 \mathrm{mg}$ twice daily by mouth was added. Three months after discharge, she was asymptomatic on mycophenolate mofetil 1,500 mg twice per day and prednisone $20 \mathrm{mg} /$ day but had developed mild essential hypertension treated with losartan $25 \mathrm{mg} /$ day. Her blood pressure on her last clinic visit was 113/75. Her 3-month follow-up labs showed improvement of the serum creatinine to $1.38 \mathrm{mg} / \mathrm{dL}$, estimated glomerular filtration rate $45 \mathrm{~mL} / \mathrm{min} / 1.73$ (normal >59), and random protein/creatinine $374 \mathrm{mg} / \mathrm{g}$ creatinine.

\section{Results}

This case is notable for three reasons. First, atypical anti-GBM nephritis is extremely rare, with less than 30 cases reported, and consequently requires a high level of clinical suspicion to diagnose [4]. The disease process is a result of autoantibodies to alpha-3 chain of type IV collagen in the basement membrane of the glomerulus [7]. Around 50\% of cases will also have lung involvement and that condition is known as Goodpasture's disease [8]. The features of the atypical presentation of anti-GBM disease include absence of both the lung involvement and undetectable circulating anti-GBM antibody. The lack of detectable offending antibodies is curious and there are several listed theories. Two possible explanations include the pathogenic antibodies produced by the patient are directed against an antigen other than the basement membrane collagen, or secondly, there is lower or transient antibody production making it undetectable by standard tests [9].

Regardless of the cause, however, using positive serologic antibody screening is not possible to rule in the disease. In fact, serologic testing is not completely reliable to make a rapid diagnosis. Since early diagnosis of the disease is important to begin treatment that could maintain renal function, an aggressive position on early renal biopsy is prudent. That is what happened in our case. The patient originally was thought to have temporary elevation of serum creatinine secondary to nonsteroidal use, but the clinical suspicion of an alternative cause resulted in recommendation of an early renal biopsy. The biopsy findings in our case were consistent with the clinical suspicion of atypical disease.

The second reason this case is unique is the approach to treatment modification that was necessary secondary to contraindications to cyclophosphamide. She was offered the standard treatment for classic anti-GBM nephritis. This choice was considered since, to date, there is no standardized therapeutic protocol for the treatment of atypical anti-GBM nephritis. Most 


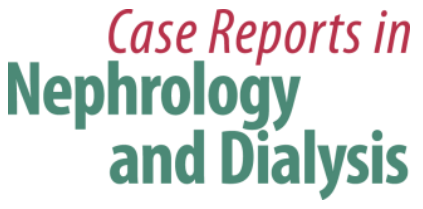

Case Rep Nephrol Dial 2019;9:8-14

DOI: $10.1159 / 000498844$

C 2019 The Author(s). Published by S. Karger AG, Basel www.karger.com/cnd

Olivier et al.: Atypical Anti-Glomerular Basement Membrane Nephritis

cases of atypical anti-GBM nephritis are managed in a similar fashion as classic anti-GBM nephritis with a combination of aggressive plasmapheresis combined with steroids and cyclophosphamide. However, there are three reports of initial management with mycophenolate mofetil rather than cyclophosphamide. One case reported complete remission and the other two reported residual renal dysfunction. The clinical outcome probably represents the stage of disease at the beginning of treatment rather than the specific agent itself [8]. Mycophenolate mofetil is an immunosuppressive agent that was initially developed as an antirejection drug and has a much more favorable toxicity profile than cyclophosphamide [6]. It has been used successfully to treat Goodpasture's disease and may be, as shown in this case, an agent that can be used as both an initial drug and a long-term immunosuppressant. How long immunosuppression should continue appears to be dependent on the level of renal disease and the antibody profile from the immunofluorescence microscopy. Those with extensive, irreversible disease should be weaned off immunosuppression as soon as possible, while those with ongoing active disease and salvageable renal function should maintain aggressive suppression [8]. Based on the clinical findings and biopsy results, we elected to recommend continued immunosuppression with mycophenolate mofetil while following her labs. Another drug that has been used to treat anti-GBM disease is rituximab. When it became apparent that the cyclophosphamide was no longer an option, our patient was counseled about this agent but declined. Rituximab is an antineoplastic agent that is reported to be useful in the treatment of anti-GBM nephritis, but there are serious reported reactions to the drug [10].

The third unique feature of this case is the antibody profile from the immunofluorescence microscopy. We found 2+ linear staining along the GBM with antibodies against IgG and kappa. All other stains including lambda were negative and there was no significant extraglomerular staining. Additional sections were incubated with antibodies specific for the subclasses of IgG, revealing $2+$ linear staining with antibodies against IgG1 and no significant staining with antibodies against IgG2, lgG3, or lgG4. This is unusual because IgG1-kappa anti-GBM nephritis has been reported only three previous times [9]. Two of the reported IgG1-kappa anti-GBM nephritis cases were treated initially with cyclophosphamide and two were relapsing $[4,10]$. There were no data on the initial treatment regimen or pathogenicity for the third case [4], and our case would be the fourth. Although data are limited, atypical IgG1-kappa anti-GBM nephritis appears to be characterized as a relapsing disease [11]. This is important in determining how to chronically manage the patient. Her notable non-dialysis-dependent level of renal involvement and her antibody profile from the immunofluorescence microscopy suggests she should be offered long-term mycophenolate mofetil both as a primary agent and as a steroid-sparing agent for maintenance immunosuppression.

\section{Conclusion}

This report describes a case of atypical IgG1-kappa anti-GBM nephritis that was diagnosed early by renal biopsy. The treatment was modified secondary to toxicity and early follow-up indicates there is no relapse yet. Early renal biopsy is critical in this condition. Determining the method of chronic management and length of treatment should reflect the likelihood of relapse. 


\section{Case Reports in and Dialysis}

Case Rep Nephrol Dial 2019;9:8-14

DOI: $10.1159 / 000498844$

(c) 2019 The Author(s). Published by S. Karger AG, Basel www.karger.com/cnd

Olivier et al.: Atypical Anti-Glomerular Basement Membrane Nephritis

\section{Acknowledgements}

No acknowledgements, financial supports, or grants to report.

\section{Statement of Ethics}

The authors have no ethical conflicts to disclose.

\section{Disclosure Statement}

There are no potential conflicts of interest for any of the authors with products or techniques discussed in the paper.

\section{Funding Sources}

There is no funding information to report.

\section{Author Contributions}

Study design: A; data collection: B; statistical analysis: C; data interpretation: D; manuscript preparation: $\mathrm{E}$; literature search: $\mathrm{F}$; funds collection: $\mathrm{G}$ (no funds were collected).

Maxim Olivier: A, B, D, E, F; Harold Watson: B, F; Danielle Lee: A, F; Viresh Mohanlal: B, D, F; Mario Madruga: B, D, E; Steve J. Carlan: A, E, F.

\section{References}

1 Salama AD, Levy JB, Lightstone L, Pusey CD. Goodpasture's disease. Lancet 2001;358:917-9.

2 Cui Z, Zhao MH, Xin G, Wang HY. Characteristics and prognosis of Chinese patients with anti-glomerular basement membrane disease. Nephron Clin Pract. 2005;99(2):c49-55.

3 Troxell ML, Houghton DC. Atypical anti-glomerular basement membrane disease. Clin Kidney J. 2016 Apr;9(2):211-21.

4 Nasr SH, Collins AB, Alexander MP, Schraith DF, Herrera Hernandez L, Fidler ME, et al. The clinicopathologic characteristics and outcome of atypical anti-glomerular basement membrane nephritis. Kidney Int. 2016 Apr;89(4):897-908.

5 Levy JB, Turner AN, Rees AJ, Pusey CD. Long-term outcome of anti-glomerular basement membrane antibody disease treated with plasma exchange and immunosuppression. Ann Intern Med. 2001 Jun;134(11):1033-42.

6 Mori M, Nwaogwugwu U, Akers GR, McGill RL. Anti-glomerular basement membrane disease treated with mycophenolate mofetil, corticosteroids, and plasmapheresis. Clin Nephrol. 2013 Jul;80(1):67-71.

7 Pedchenko V, Bondar O, Fogo AB, Vanacore R, Voziyan P, Kitching AR, et al. Molecular architecture of the Goodpasture autoantigen in anti-GBM nephritis. N Engl J Med. 2010 Jul;363(4):343-54.

8 L'Imperio V, Ajello E, Pieruzzi F, Nebuloni M, Tosoni A, Ferrario F, et al. Clinicopathological characteristics of typical and atypical anti-glomerular basement membrane nephritis. J Nephrol. 2017 Aug;30(4):503-9.

9 Syeda UA, Singer NG, Magrey M. Anti-glomerular basement membrane antibody disease treated with rituximab: A case-based review. Semin Arthritis Rheum. 2013 Jun;42(6):567-72.

10 Bahrainwala JZ, Stokes MB, Hannani AK, Hogan JJ. Atypical antiglomerular basement membrane disease with IgG1-к staining. Kidney Int Rep. 2016 Aug;2(1):80-3.

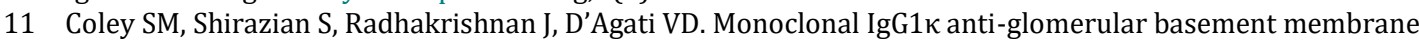
disease: a case report. Am J Kidney Dis. 2015 Feb;65(2):322-6. 


\section{Case Reports in Nephrology and Dialysis}
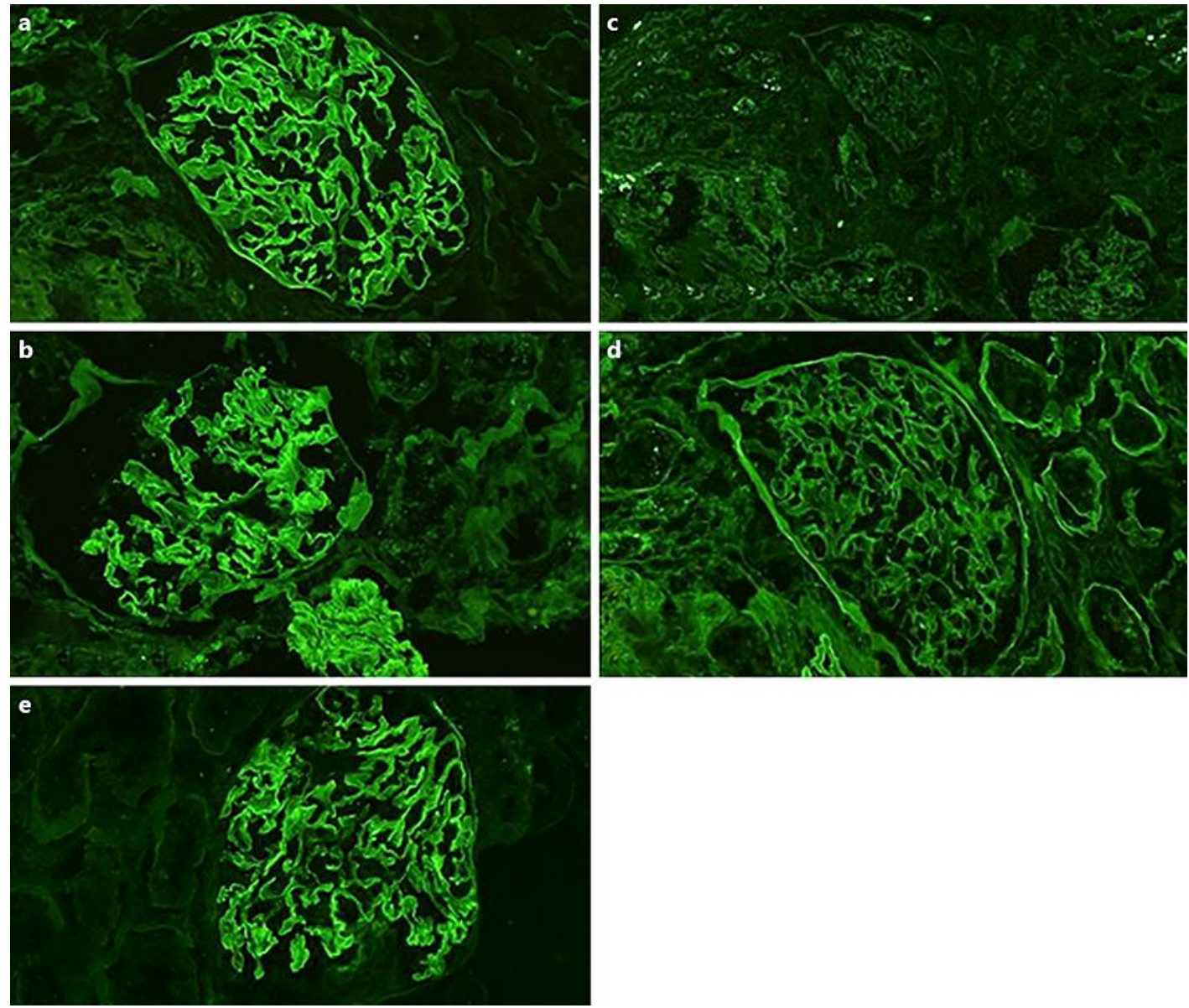

Fig. 1. Immunofluorescence shows $2+$ linear stating along the glomerular basement membrane with antibodies against IgG (a) and kappa (b). All other stains including lambda (c) and albumin (d) were negative. IgG1 subclass shows 2+ linear staining of glomerular basement membrane (e). Staining for IgG subclasses IgG2, IgG3, and IgG4 was negative (not shown). 


\section{Case Reports in Nephrology and Dialysis}

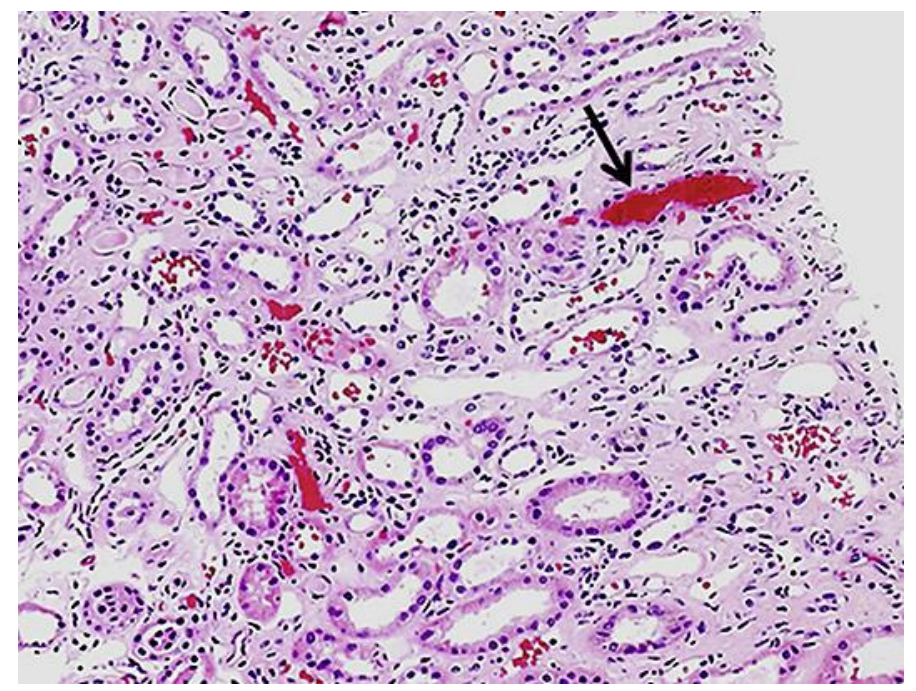

Fig. 2. H\&E staining on light microscopy shows RBC casts of varying ages in the tubular lumens.

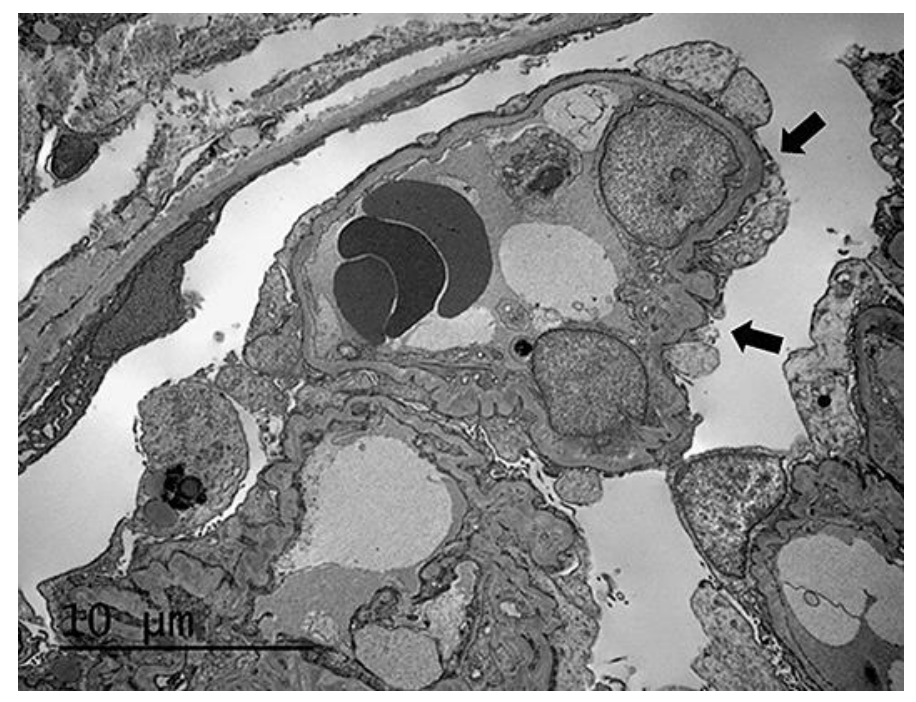

Fig. 3. Electron microscopic examination of glomerulus reveals widespread effacement of the foot processes (black arrows) and absence of any immune complex electron dense deposits. 